\title{
Le récepteur
} dopaminergique $D_{3}$ i nouvelle cible pour un traitement

> La schizophrénie est une maladie complexe et grave, qui touche $0,5 \%$ à $1,0 \%$ de la population. Cette maladie, qui s'installe dès l'adolescence (15-25 ans), est progressive et souvent irréversible, avec un coût social très élevé. Les sympamélioré de la schizophrénie

Mark J. Millan tômes positifs, comme les hallucinations, sont assez bien contrôlés par divers « antipsychotiques », tandis que les troubles cognitifs et déficitaires restent difficiles à traiter. Les antipsychotiques possèdent des profils d'interactions avec des récepteurs très différents, mais interagissent tous avec les voies dopaminergiques dont l'activité est perturbée chez les patients souffrant de schizophrénie. La dopamine agit par l'intermédiaire de cinq classes de récepteurs, ce qui représente une palette étendue pour l'élaboration de nouvelles approches thérapeutiques. Des résultats expérimentaux récents suggèrent que les récepteurs de soustype $D_{3}$ sont impliqués dans l'étiologie de la schizophrénie, et les premières études cliniques utilisant des antagonistes $D_{3}$ ont été récemment mises en route pour évaluer cette hypothèse. <

\section{La schizophrénie: une maladie dévastatrice au coût social élevé}

La schizophrénie est une maladie grave qui aboutit à une désorganisation de la personnalité, une perturbation des rapports familiaux, un isolement social et une incapacité à travailler [1]. D'une façon générale, cette maladie débute à l'adolescence (entre 15 et 25 ans). Progressive et souvent (mais pas toujours) irréversible, elle est ponctuée de crises abruptes. Bien que la schizophrénie soit relativement rare, son début précoce, sa chronicité, sa morbidité importante (un patient sur quatre fera au moins une tentative de suicide), l'inaptitude des patients à travailler et la nécessité de longs séjours hospitaliers font que cette maladie a un coût social très élevé [2]. La schizophrénie présente plusieurs dimen-

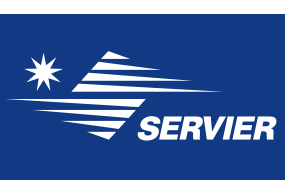

IDR Servier, Départment de Psychopharmacologie, 125 , chemin de ronde, sions psychopathologiques pouvant se regrouper en 78290 Croissy-sur-Seine, France. trois constellations diagnostiques (Tableau I). En plus de la dichotomie entre les symptômes positifs (productifs) et négatifs (déficitaires), les troubles cognitifs sont de plus en plus évoqués en raison de leur association avec les problèmes fonctionnels $[1,3,4]$. Ces atteintes cognitives, qui apparaissent relativement tôt chez les jeunes malades, peuvent être à l'origine (rôle déclencheur) des autres signes de la maladie.

\section{Traitement de la schizophrénie: un besoin thérapeutique important}

Une analyse des antipsychotiques disponibles actuellement (Tableau II) montre qu'il reste toujours des carences dans le traitement de la schizophrénie [4-6]. $\varepsilon n$ termes d'amélioration clinique (qui permettrait la réintégration familiale et sociale du patient ainsi qu'une reprise du travail), les objectifs principaux pour de nouveaux traitements sont d'améliorer la qualité de la rémission et traiter davantage de patients résistants, d'agir plus efficacement contre les symptômes déficitaires, d'obtenir des effets thérapeutiques nets contre les symptômes cognitifs et d'éviter les effets secondaires néfastes majeurs. Aujourd'hui, la seule stratégie pour atteindre ces objectifs est la recherche de nouveaux médicaments plus efficaces et mieux tolérés. II est irréaliste d'imaginer que l'on pourra trouver la molécule répondant parfaitement à ce cahier des 
charges et convenant à chaque patient: pour cette raison, il est essentiel d'explorer diverses hypothèses de travail.

\section{Identification des cibles potentielles de nouveaux antipsychotiques}

Le génome humain pourrait être une source importante de nouveaux antipsychotiques car de nombreux gènes ont été associés à un risque élevé de schizophrénie. Néanmoins, pour la plupart de ces gènes, les contributions sont mineures et restent à confirmer [7, 8]. II faut également rappeler que les gènes ou les protéines dont le dysfonctionnement serait en cause dans la schizophrénie ne sont pas forcément ceux que l'on doit cibler pour son traitement. Le défi principal, aujourd'hui, reste donc l'identification des cibles thérapeutiques permettant une meilleure prise en charge de la schizophrénie. Dans la recherche de ces cibles, diverses approches complémentaires, autres que génétiques, se sont révélées riches d'enseignements. Les techniques de plus en plus performantes d'imagerie ont montré, chez les schizophrènes, des changements structurels marqués dans le thalamus, l'hippocampe, le cortex et d'autres structures cérébrales [9, 10]. Plus récemment, ces méthodes ont été appliquées à l'identification des modulateurs (neurotransmetteurs, neurotrophines, signaux intracellulaires...) dont l'activité est modifiée soit par la schizophrénie elle-même, soit par le traitement par les antipsychotiques $[9,10]$. Ces approches se font en parallèle avec des études neurochimiques du cerveau des schizophrènes. Par ailleurs, les études neurochimiques et comportementales chez l'animal sont indispensables pour caractériser les systèmes neuronaux (et gliaux) modulés soit par les antipsychotiques, soit par les composés induisant les états psychotiques tels que la cocaïne (psychostimulant), la mescaline (hallucinogène) et surtout la phencyclidine [11, 12]. La phencyclidine (plus connue des toxicomanes sous le nom de «poussière d'ange ») est la seule substance qui reproduise chaque dimension de la schizophrénie chez le sujet sain. Elle se comporte comme un antagoniste non compétitif des récepteurs glutamatergiques de sous-type «NMDA » [12]. En accord avec ces observations (et d'autres), les récepteurs NMDA sont actuellement une cible privilégiée dans la découverte de nouveaux antipsychotiques $[12,13]$. D'une façon intéressante, certains effets comportementaux de la phencyclidine semblent mettre en jeu des mécanismes sérotoninergiques, notamment une suractivation des récepteurs $5-\mathrm{HT}_{2 \mathrm{~A}}$ au niveau limbique [14]. Ces résultats soulignent I'hypothèse selon laquelle un blocage des sites $5-\mathrm{HT}_{2 \mathrm{~A}}$ serait à l'origine des profils «atypiques » cliniques de la clozapine et peut-être d'autres antipsychotiques de nouvelle génération [15] qui, par rapport aux neuroleptiques, présentent une fenêtre thérapeutique améliorée entre effets bénéfiques et effets secondaires moteurs (Tableau II). Concernant la sérotonine, les propriétés agonistes $5-\mathrm{HT}_{1 \mathrm{~A}}$ et $5-\mathrm{HT}_{2 \mathrm{C}}$, ou antagonistes $5-\mathrm{HT}_{6}$, ont également suscité l'intérêt en tant que mécanismes d'action potentiels pour de nouveaux antipsychotiques. D'autres cibles nouvelles devraient également être citées: les cannabinoïdes (antagonistes des sites $C_{1}$ ), la substance $P$ (antagonistes des neurokinines $\mathrm{NK}_{3}$ ), la neurotensine (agonistes $\mathrm{NT}_{1}$ ), l'acétylcholine (agonistes nicotiniques et muscariniques) et la noradrénaline (antagonistes $\alpha_{2}$ ). Toutes ces hypothèses sont d'un grand intérêt théorique [13, 15-17]. Néanmoins, il n'existe aucune preuve clinique que les médicaments agissant principalement sur ces cibles soient vraiment en mesure de traiter efficacement la schizophrénie.

Aujourd'hui, nous ne pouvons citer qu'un seul et unique transmetteur dont l'implication dans l'étiologie et surtout le traitement de la schizophrénie soit irréfutable: il s'agit de la dopamine (DA), cible impliquée dans les effets de tout antipsychotique utilisé actuellement [18, 19]. Pour comprendre l'importance de la DA dans la pathogénie et le traitement de la schizophrénie, il faut tenir compte des diverses voies neuronales dopaminergiques ainsi que de la multiplicité des récepteurs impliqués dans ses effets.

\begin{tabular}{lll}
\hline Symptômes positifs & Symptômes négatifs & Atteintes cognitives \\
Hallucinations & Retrait social & Mémoire de travail \\
Illusions & Apathie/mutisme & Attention/vigilance \\
Agitation & Émoussement affectif & Cognition verbale \\
Idées délirantes & Désorganisation de la pensée, du langage & Cognition sociale \\
\hline
\end{tabular}

Tableau I. La schizophrénie, une symptomatologie complexe. Les signes positifs sont les mieux connus et consistent en hallucinations auditives (le sujet entend des voix), en l'impression d'être téléguidé (le sujet doit obéir à autrui), en idée de persécution par des êtres extérieurs, et en un état d'irritation et d'agitation extrême. Néanmoins, à long terme, à part les crises aiguës, ce sont les signes négatifs et cognitifs qui sont les plus difficiles à vivre - et les plus difficiles à traiter. Ils sont susceptibles de progressivement conduire à un isolement social et à une incapacité à travailler.

\section{Les voies \\ dopaminergiques dans l'étiologie de la schizophrénie}

\section{Modifications} pathologiques des voies dopaminergiques

Les voies mésolimbiques issues de l'aire tegmentale ventrale projettent vers le noyau accumbens et d'autres structures limbiques. Les études d'ima- 
gerie entreprises chez des patients souffrant de schizophrénie, ainsi que les études cliniques et expérimentales effectuées avec des composés inducteurs des états psychotiques, montrent qu'une hyperactivité et une hypersensibilité de ces voies sont à l'origine des symptômes positifs de la schizophrénie [10, 18-22]. En revanche, les voies mésocorticales dopaminergiques qui innervent le cortex frontal ne sont pas suractivées chez les patients souffrant de schizophrénie: leur activité serait plutôt diminuée, en accord avec les études montrant une atteinte fonctionnelle et structurelle du cortex frontal dans la schizophrénie $[10,15,23]$. Cette «hypofrontalité » est impliquée dans les symptômes négatifs et cognitifs de la maladie. Par ailleurs, l'activité des neurones dopaminergiques nigrostriés n'est pas modifiée chez les schizophrènes. Cette voie, qui relie la substance noire et le striatum, joue un rôle important dans la coordination de la fonction motrice $[19,24]$. De même, les faisceaux hypothalamo-hypophysaires dopaminer- giques, inhibiteurs de la sécrétion de prolactine, ne sont pas affectés. Bien évidement, les conséquences fonctionnelles d'une modulation par les antipsychotiques de l'activité de ces réseaux dopaminergiques sont très différentes (voir ci-dessous).

\section{Multiples récepteurs pour la DA}

La DA joue un rôle majeur dans le contrôle de l'humeur, de la cognition et de l'activité motrice, ces fonctions étant modifiées chez les schizophrènes, et par l'administration d'antipsychotiques [16, 17, 20 , 25]. Les effets de la DA sont exprimés par l'intermédiaire de diverses classes de récepteurs qui sont réparties en deux familles: $D_{1} / D_{5}$ et $D_{2} / D_{3} / D_{4}$, couplées d'une façon positive et négative, respectivement, à l'adénylate cyclase (Figure 1) [26]. Les récepteurs $D_{2}$ et $D_{3}$ se situent au niveau post-synaptique ainsi que présynaptique. Ces derniers agissent en tant qu'autorécepteurs inhibiteurs de la transmission dopaminer-

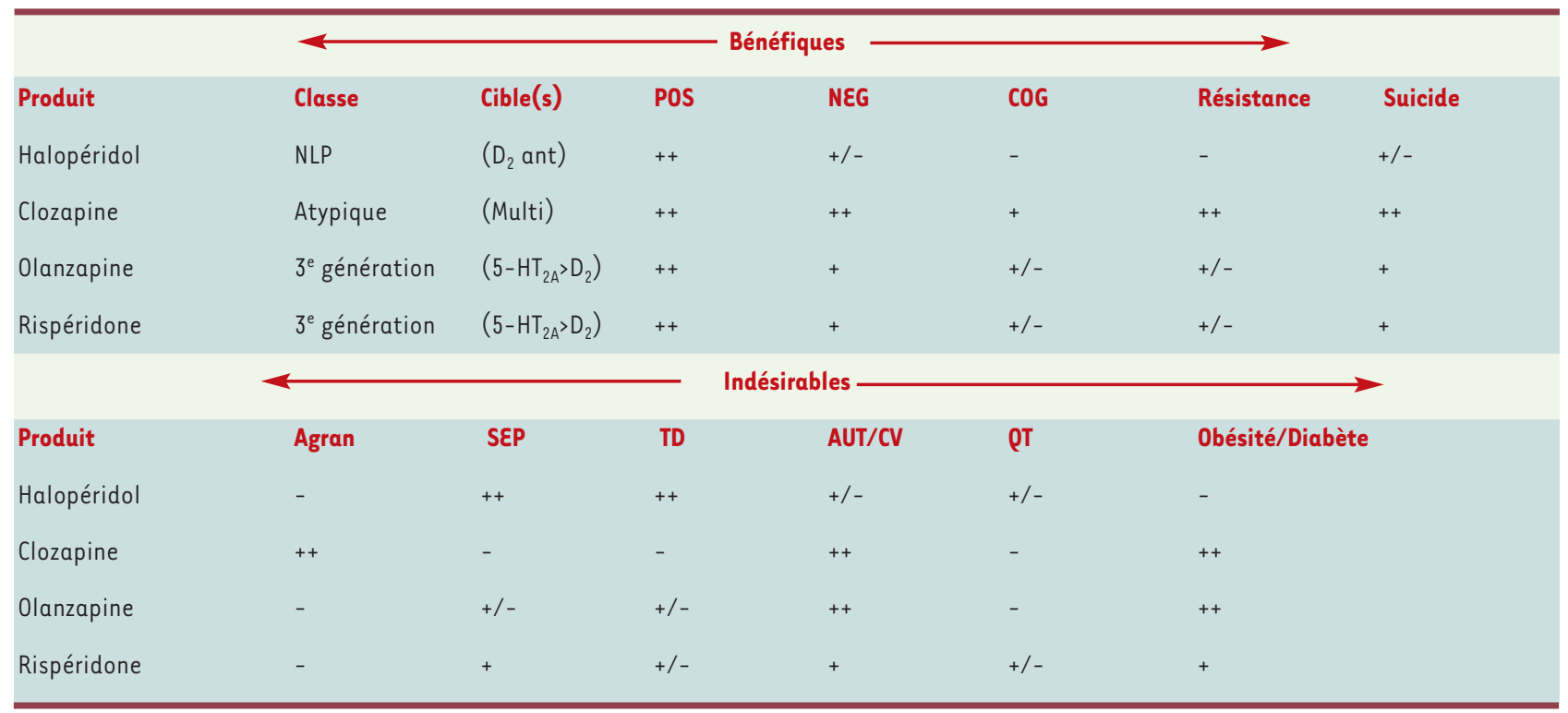

Tableau II. Profils thérapeutiques de l'halopéridol, de la clozapine et de deux antipsychotiques de $3^{e}$ génération (olanzapine et rispéridone). Les neuroleptiques sont efficaces contre les signes positifs, mais au prix d'effets extrapyramidaux moteurs et endocriniens (prolactinémie) induits, respectivement, aux niveaux strié et hypophysaire. Ils n'améliorent que faiblement les symptômes déficitaires et cognitifs. Ce sont de puissants antagonistes des récepteurs $D_{2}$ et $D_{3}$. La clozapine est active chez de nombreux patients réfractaires aux neuroleptiques et diminue le risque de suicide. La notion d' «atypique » fait référence à sa capacité de contrôler les symptômes positifs sans provoquer des effets extrapyramidaux. Par ailleurs, la clozapine est plus active que les neuroleptiques sur les symptômes négatifs et cognitifs, bien que son efficacité procognitive reste mitigée en raison de ses propriétés anti-muscariniques, anti-adrénergiques et anti-histaminergiques qui induisent, en plus, des effets autonomes/cardiovasculaires et métaboliques indésirables. Les mécanismes à l’origine des effets bénéfiques de la clozapine ne sont pas connus. Outre ses effets dopaminergiques, on peut évoquer ses propriétés glutamatergiques indirectes (facilitation), ainsi qu'antagonistes $5-\mathrm{HT}_{2 \mathrm{~A}} / 5-\mathrm{HT}_{2 \mathrm{C}}$ et agonistes $5-\mathrm{HT}_{1 \mathrm{~A}}$. Une préférence nette pour les récepteurs $5-\mathrm{HT}_{2 \mathrm{~A}}$ versus $\mathrm{D}_{2}$ est caractéristique de l'olanzapine et de la rispéridone ainsi que d'autres antipsychotiques de troisième génération, tels que la quétiapine et la ziprasidone. NLP: neuroleptique; ant: antagoniste; Multi : multirécepteur; POS: symptômes positifs; NEG: symptômes négatifs; COG: symptômes cognitifs; Agran: agranulocytose; SEP: syndrome extrapyramidal; TD: dyskinésie tardive; QT: allongement des ondes QT cardiaques; AUT/CV: effets autonomes/cardiovasculaires. ++: marqué; +: bien documenté, mais moins marqué; +/-: variable et peu marqué; - : absent. 
gique. Un important défi des 10 dernières années, l'élucidation des rôles fonctionnels des cinq classes de récepteurs dopaminergiques, a nécessité l'élaboration de différentes approches expérimentales complémentaires: antagonistes sélectifs, sondes antisens et souris génétiquement modifiées [20,25]. Ces stratégies de recherche ont également été appliquées à l'exploration d'une question fondamentale, la signification des cinq classes de récepteurs dopaminergiques dans le contrôle des états psychotiques. Dans cette optique, avant une discussion détaillée des récepteurs $D_{3}$, il est intéressant d'évoquer quelques concepts dopaminergiques actuellement étudiés dans le cadre de la mise en œuvre de meilleurs antipsychotiques.

\section{De nouveaux concepts dopaminergiques dans le traitement de la schizophrénie}

Certains experts considèrent que les propriétés bénéfiques de toutes les classes d'antipsychotiques (y compris la clozapine) s'expliquent seulement par leurs interactions avec les récepteurs $D_{2}$ [16]. Les différences en termes de fenêtre thérapeutique (doses contrôlant les symptômes positifs versus celles induisant des effets extrapyramidaux [SEP] moteurs)

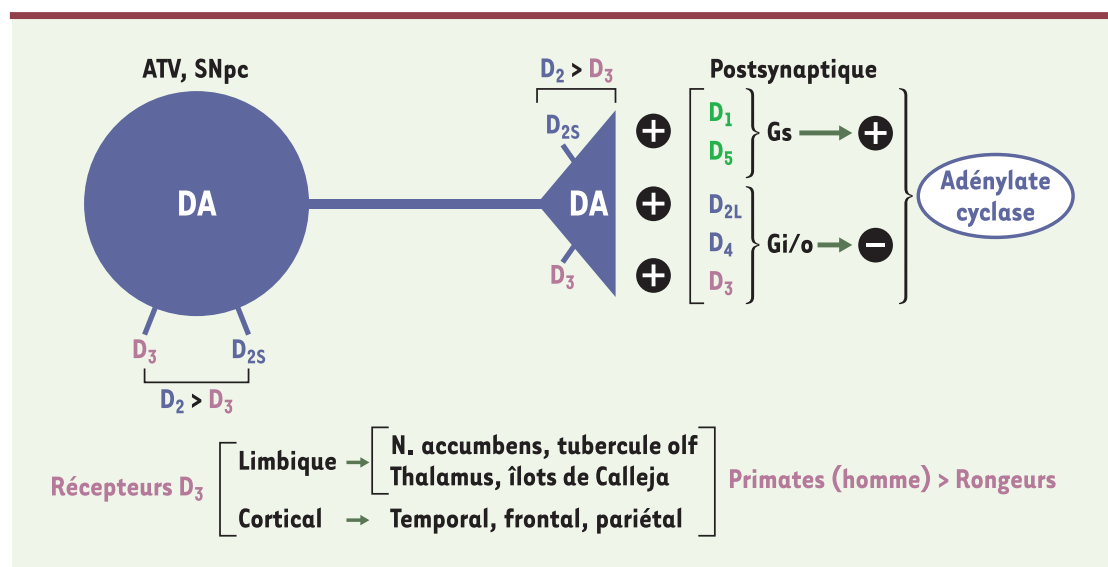

Figure 1. Localisation neuronale des récepteurs $D_{3}$ par rapport à celle d'autres classes de récepteurs dopaminergiques. Les voies mésolimbiques issues de l'aire tegmentale ventrale (ATV) et de la substantia nigra pars compacta (SNpc) projettent vers le noyau accumbens et d'autres structures limbiques. Sur le plan neuronal, les récepteurs $D_{3}$ se trouvent à la fois au niveau postsynaptique et au niveau présynaptique, à l'instar des récepteurs $D_{2}$ dont les isoformes «short » (S) et «long » (L) sont concentrés pré- et postsynaptiquement, respectivement. La densité des autorécepteurs $D_{3}$ est inférieure à celle des récepteurs $D_{2}$ qui exercent également une influence inhibitrice sur l'activité des voies dopaminergiques (DA). Par l'intermédiaire des sous-classes de protéines $\mathrm{G}$ différentes (Gs, Gi, Go), les récepteurs dopaminergiques modulent l'activité du messager secondaire, l'adénylate cyclase. À la différence d'autres classes de récepteurs dopaminergiques, les récepteurs $D_{3}$ sont localisés préférentiellement dans les structures corticolimbiques. La densité des récepteurs $D_{3}$ est plus importante chez l'homme et chez d'autres primates que chez le rat. seraient le reflet de leurs façons différentes de se fixer aux récepteurs $D_{2}$. Ainsi, les composés peu affins comme la clozapine, qui de plus se fixent de manière «faible», auraient des fenêtres thérapeutiques plus importantes. Cette hypothèse a le mérite de réaffirmer l'importance des mécanismes dopaminergiques dans fait abstraction de tout récepteur autre que $D_{2}$ pour expliquer le profil thérapeutique de la clozapine, ce qui n'est pas entièrement satisfaisant ! De plus, comme les protagonistes de cette hypothèse l'admettent volontiers, elle ne différencie pas le rôle des sites $D_{2}$ et $D_{3}$ comme cible dans le traitement de la schizophrénie [16]. En outre, les radioligands utilisés aujourd'hui dans les études d'imagerie pour marquer les récepteurs $« D_{2} \gg$ chez l'homme ne discriminent pas les différents sous-types $D_{2}, D_{3}$ et $D_{4}[10,18,20]$.

De nombreux projets de recherches se focalisent actuellement sur les propriétés agonistes $D_{1}$ dans l'espoir d'améliorer la fonction cognitive des schizophrènes. Cette notion est ancrée dans les effets procognitifs des agonistes $D_{1}$ dans les modèles comportementaux chez les rongeurs $[25,27]$. Les agonistes $D_{1}$ facilitent la transmission cholinergique qui joue un rôle critique dans les processus cognitifs [27]. En revanche, en raison d'un éventuel phénomène d'épuisement, il reste à démontrer que les effets bénéfiques des agonistes $D_{1}$ se manifestent à long terme.

Idéalement, il faudrait combiner l'activité agoniste $D_{1}$ avec un blocage des récepteurs $D_{2} / D_{3}$ pour bénéficier également des effets thérapeutiques contre les symptômes positifs.

Finalement, les agonistes partiels $D_{2}$ représentent un concept intéressant bien illustré par l'aripiprazole, antipsychotique récemment mis sur le marché [28]. Ce composé interagit avec divers sites monoaminergiques, mais a la particularité de se comporter comme un agoniste faible des récepteurs $D_{2}$. De ce fait, l'aripiprazole stimule les sites présynaptiques $D_{2}$ très sensibles et inhibiteurs de la libération de la DA, alors qu'il bloque les récepteurs postsynaptiques peu sensibles $D_{2}$ [28]. L'aripiprazole possède donc un double mécanisme pour «normaliser» I'hyperactivité des voies dopaminergiques mésolimbiques. II est actif contre les symptômes positifs sans pour autant provoquer d'effets SEP. Néanmoins, il faudra attendre quelques années d'utilisation clinique pour avoir la confirmation de son efficacité et de sa bonne tolérance. De plus, l'efficacité 
de l'aripiprazole contre les symptômes déficitaires et cognitifs reste limitée [28].

\section{Les récepteurs dopaminergiques $D_{3}$ : pertinence dans le traitement de la schizophrénie}

Bien que la structure et les voies de couplage des récepteurs $D_{3}$ soient proches de celles des sites $D_{2}$, leur distribution et leurs rôles fonctionnels diffèrent d'une manière significative dans le contrôle des états psychotiques.

\section{Induction d'effets SEP moteurs}

À la différence des sites $D_{2}$, les récepteurs $D_{3}$ se trouvent majoritairement dans des structures corticales et limbiques [17]. Leur densité est faible dans le striatum et l'hypophyse. Cette organisation suggère qu'un blocage sélectif des récepteurs $D_{3}$ ne devrait pas induire d'effets SEP moteurs, ni d'effets endocriniens, interprétation appuyée par les études expérimentales effectuées avec des antagonistes $D_{3}$ sélectifs, les sondes d'antisens dirigées contre les sites $D_{3}$ et les souris génétiquement privées de sites $D_{3}[17,20,25$, 29]. Ces observations sont d'autant plus intéressantes que l'inactivation des sites $D_{3}$ s'oppose aux effets moteurs gênants des antagonistes $D_{2}[30]$. Ainsi, un blocage préférentiel des récepteurs $D_{3}$ versus $D_{2}$ par un antagoniste devrait minimiser l'impact SEP.

\section{Contrôle des symptômes déficitaires} L'absence d'effets SEP avec un antagoniste $D_{3}$ serait importante dans le traitement des symptômes déficitaires qui sont aggravés par les effets moteurs perturbateurs dus aux propriétés antagonistes $D_{2}$ des neuroleptiques. De plus, un blocage des récepteurs $D_{2}$ exerce des effets néfastes sur l'humeur, ce qui exacerbe également les symptômes négatifs, tandis que les antagonistes $D_{3}$ sont dépourvus de telles actions délétères [31]. Les symptômes déficitaires sont difficiles à modéliser chez l'animal, mais un élément accessible est l'isolement social. À la différence des antagonistes $D_{2}$, les antagonistes $D_{3}$ favorisent l'interaction sociale chez les rongeurs $[32,33]$. Une autre approche pour appréhender l'influence des antagonistes $D_{3}$ sur les signes déficitaires consiste à comparer leurs effets à ceux de la clozapine. En effet, cette dernière induit des effets distincts sur les facteurs de transcription et d'autres gènes cérébraux, dont certains sont mimés par les antagonistes $D_{3}$ [34]. De plus, ces effets de la clozapine sont absents chez les souris génétiquement privées de récepteurs $D_{3}[34]$.

\section{Contrôle des symptômes cognitifs}

Un blocage des récepteurs $D_{2}$ 'exerce aucune influence bénéfique sur la fonction mnésique chez l'animal et peut même la compromettre, en accord avec l'incapacité des neuroleptiques à améliorer les symptômes cognitifs chez les schizophrènes $[3,4]$. En revanche, les études récentes effectuées sur des souris privées de sites $D_{3}$ révèlent une amélioration de certaines facettes de la fonction cognitive [35]. De plus, nous avons montré un impact favorable des antagonistes $D_{3}$ sur la fonction cognitive dans un modèle de mémoire de travail (reconnaissance sociale) [36] qui est fortement perturbée chez les schizophrènes [3, 4]. Cette observation est d'autant plus intéressante que les

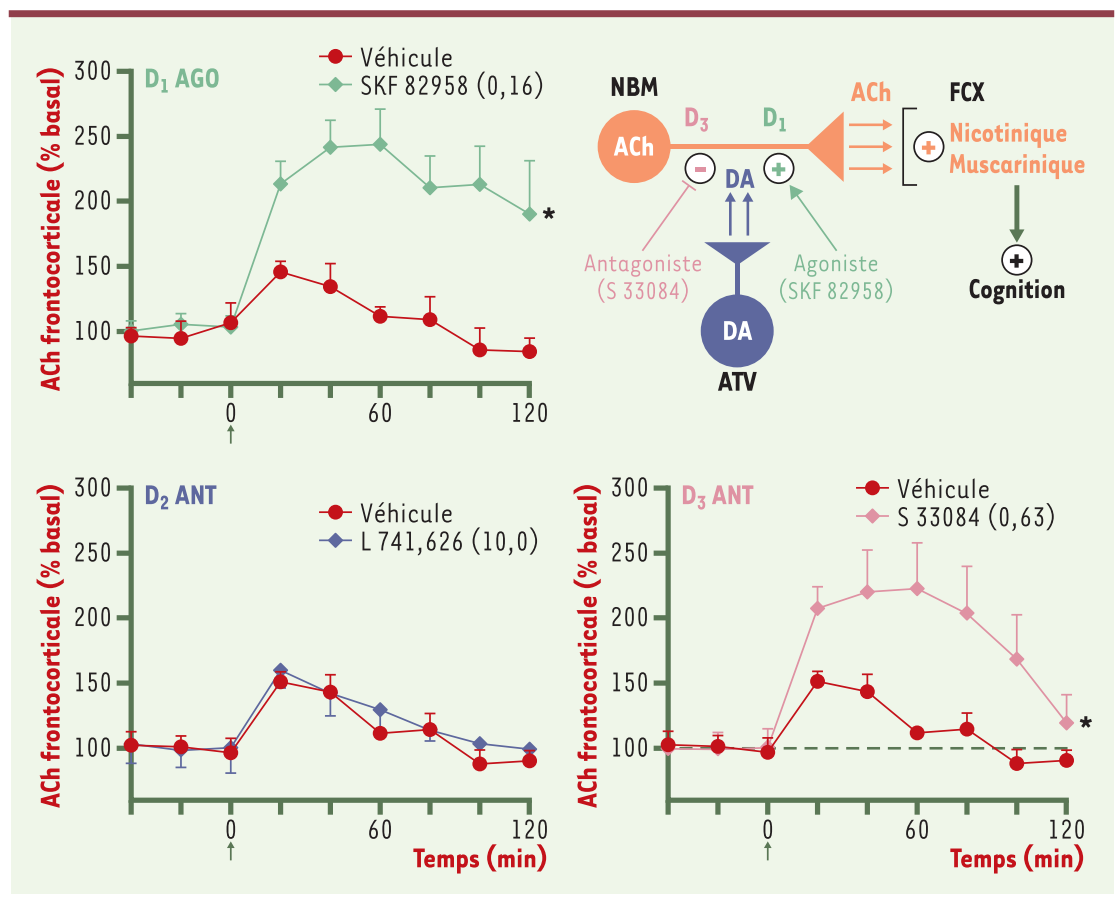

Figure 2. Influence des récepteurs $D_{3}$ sur la libération de l'acétylcholine (ACh) mesurée dans le cortex frontal de rats vigiles. L'ACh a été mesurée (dans le laboratoire de l'auteur) en microdialyse couplée à une détection électrochimique. Le taux d’ACh est exprimé en pourcentage des valeurs basales (avant injection s.c.) qui sont définies comme 100\%. Les doses des composés sont exprimées en $\mathrm{mg} / \mathrm{kg}$ du produit. * $\mathrm{p}<0,01$ par rapport au véhicule (solvant). À l'instar de l'agoniste sélectif $D_{1}$, le SKF 82958, et à la différence de l'antagoniste $D_{2}$, le L 741,626, l'antagoniste sélectif $D_{3}$, le $S 33084$, renforce la transmission cholinergique. Le schéma (en haut à droite) indique que les récepteurs $D_{1}$ et $D_{3}$ exercent une influence directe sur les neurones cholinergiques. AVT: aire ventrotegmentale; NBM: noyau basal de Meynert; FCX: cortex frontal; AGO: agoniste; ANT: antagoniste. 
antagonistes $D_{3}$, comme les agonistes $D_{1}$, augmentent la transmission frontocorticale cholinergique (Figure 2) [27, $36,37]$, substrat de la mémoire de travail perturbée chez les schizophrènes [3, 4]. Le rôle des récepteurs $D_{3}$ dans le contrôle de la fonction mnésique nécessite une exploration plus approfondie, mais il est fort possible qu'un blocage préférentiel des sites $D_{3}$ soulage les troubles cognitifs des patients schizophrènes.

\section{Contrôle des symptômes positifs}

Paradoxalement, l'efficacité potentielle des antagonistes $D_{3}$ contre les symptômes positifs de la schizophrénie semble la moins bien démontrée actuellement, dans la mesure où ils sont peu ou pas actifs dans la plupart des modèles prototypes répondant bien aux autres classes d'antipsychotiques. Ainsi, un antagoniste $D_{3}$ ne bloque pas les effets moteurs de l'amphétamine chez le rat $[17,29,32]$. Néanmoins, ces modèles animaux ont été développés spécifiquement pour la mise en évidence des propriétés antagonistes $D_{2}$ des antipsychotiques et ne sont pas forcément à l'origine de leurs effets thérapeutiques [11, 17, 29].

D'ailleurs, dans certains modèles, les antagonistes $D_{3}$ sont bien actifs [38]. Par exemple, un traitement chronique avec les antagonistes $D_{3}$ diminue l'activité électrique des neurones mésolimbiques $[17,32,33]$. En revanche, les neurones nigrostriés ne sont pas affectés, soulignant l'absence de potentiel inducteur du SEP des antagonistes $D_{3}$. Ce profil ressemble à celui de la clozapine et se démarque des neuroleptiques qui modulent à la fois les voies mésolimbiques et nigrostriées $[17,32]$. Cette ambiguïté concernant la capacité des antagonistes $D_{3}$ à modérer les symptômes positifs reste un peu troublante. En revanche, les résultats expérimentaux penchent en faveur d'une influence favorable sur les symptômes déficitaires et cognitifs. Étant donné les limitations des modèles expérimentaux, comme pour chaque nouvel antipsychotique, seuls les essais cliniques sur les patients apporteront une réponse définitive.

Une implication des récepteurs $D_{3}$ dans l'étiologie de la schizophrénie?

Étant donné l'augmentation de l'activité des voies mésolimbiques dopaminergiques chez les schizophrènes $[10,17]$, il est important de considérer une éventuelle modification du taux des récepteurs $D_{3}$ chez ces patients. En effet, la densité des récepteurs $D_{3}$ (mais non $D_{2}$ ) limbiques est élevée chez les schizophrènes [17, 39]. De surcroît, cette augmentation est normalisée après traitement par les antipsychotiques. Cette élévation du taux des sites $D_{3}$ est en accord avec des études expérimentales suggérant qu'une élévation et une réduction de l'activité des neurones dopaminergiques soient accompagnées par une up versus down régulation des récepteurs $D_{3}$, respectivement $[17,24]$. Ces résultats chez les patients schizophrènes sont également soutenus par l'augmentation comparable du taux des récepteurs $D_{3}$ limbiques observée chez les cocaïnomanes [17, 24]. Ainsi, une suractivation des voies mésolimbiques dopaminergiques impliquées dans les symptômes positifs pourrait être associée avec une activité accrue des sites $D_{3}$. Diverses études ont examiné une association potentielle entre la schizophrénie et la présence d'un polymorphisme du gène codant pour les récepteurs $D_{3}$. II s'agit de la substitution d'une sérine par une glycine (Ser9Gly) qui aurait comme conséquence une affinité plus importante pour la DA. La majorité de ces études suggère effectivement une association positive entre ce polymorphisme et la schizophrénie, mais de nombreux rapports négatifs ne permettent pas de conclusions définitives $[40,41]$. Une association éventuelle entre les récepteurs $D_{3}$, seuls ou en interaction avec d'autres gènes, et la schizophrénie nécessite donc des analyses plus complètes. pine; Aripip: aripiprazole. 
Les antagonistes $D_{3}$, un concept innovant pour améliorer le traitement de la schizophrénie

\section{Les antagonistes $D_{3}$ sélectifs}

À partir des arguments présentés dans les paragraphes précédents, une évaluation clinique des effets d'un blocage des récepteurs $D_{3}$ chez les schizophrènes est entièrement justifiée. Divers antagonistes $D_{3}$ très sélectifs ont été décrits, dont certains sont en développement clinique, sans pour autant que les résultats thérapeutiques soient déjà disponibles $[29,32,33,42]$. Bien que I'on puisse être assez optimiste concernant leur influence sur les signes déficitaires et cognitifs ainsi que leur absence d'impact SEP, la plus grande interrogation avec de tels ligands sélectifs $D_{3}$ porte sur leur influence potentielle sur les symptômes positifs.

\section{Les antagonistes $D_{3}$ optimisés, un concept novateur} Ces considérations nous ont conduit à élaborer un concept complémentaire: les antagonistes $D_{3}$ « optimisés », qui montrent une préférence nette, mais non une sélectivité absolue, pour les récepteurs $D_{3}$ versus $D_{2}[43$, 44]. Ce concept doit en principe permettre l'exploration clinique de deux hypothèses complémentaires. Premièrement, sur une gamme de doses faibles, l'utilité d'un blocage exclusif des récepteurs $D_{3}$ peut être évaluée. Deuxièmement, sur une gamme de doses plus élevées, les effets d'un blocage préférentiel des sites $D_{3}$ versus $D_{2}$ peuvent être explorés. Si un antagonisme sélectif des récepteurs $D_{3}$ se montre peu efficace vis-à-vis des signes positifs, un blocage au moins partiel des sites $D_{2}$ par les doses élevées devrait être efficace, tandis que l'occupation plus importante des sites $D_{3}$ versus $D_{2}$ limiterait les effets SEP moteurs et devrait garantir un bon contrôle des symptômes déficitaires et cognitifs. Concernant l'importance d'un meilleur contrôle des signes cognitifs par un blocage des récepteurs $D_{3}$, il faut signaler une initiative de la FDA (Food and Drug Administration) et du National Institute of Mental Health, aux États-Unis, qui illustre bien à quel point ce besoin thérapeutique est maintenant reconnu [45]. Pour la toute première fois, la FDA va autoriser des études cliniques consacrées exclusivement à l'influence de composés novateurs sur les symptômes cognitifs. Cette démarche prévoit d'utiliser de nouveaux médicaments soit seuls, soit en association avec d'autres classes d'antipsychotiques, contre les symptômes cognitifs, même si leur influence sur les symptômes positifs (et négatifs) est peu marquée. D'ailleurs, l'administration d'antipsychotiques procognitifs à de jeunes patients prodromaux qui présentent des atteintes cognitives pourrait éventuellement prévenir ou retarder le déve- loppement des symptômes positifs [3, 4].

\section{Le $S 33138$, antagoniste $D_{3}$ optimisé}

\section{et antipsychotique potentiel}

Actuellement, le seul composé décrit dans cette classe est le S 33138, issu de la recherche Servier [43, 44]. La découverte de ce composé repose sur les études étendues de structure-activité autour d'un squelette chimique qui au départ possédait une affinité faible, mais préférentielle pour les récepteurs $D_{3}$ versus $D_{2}$. Une collaboration étroite entre pharmacologues et chimistes a permis l'optimisation progressive des structures pour enfin atteindre le profil souhaité du S 33138. Dans divers modèles, le $S 33138$ a été systématiquement comparé aux autres antipsychotiques, ce qui a confirmé son originalité en termes de préférence nette pour les récepteurs $D_{3}$ versus $D_{2}$ (Figure 3 ) et de profil fonctionnel in vivo. Dans les modèles cellulaires et fonctionnels in vivo, le $\mathrm{S} 33138$ se comporte comme un antagoniste pur des sites $D_{3}$ et, sur une gamme de concentrations plus élevées, des sites $D_{2}$. À doses faibles, le $S 33138$ renforce la transmission cholinergique corticale, améliore la performance des rats dans des modèles de mémoire de travail et facilite l'interaction sociale. Ces résultats suggèrent une influence bénéfique sur les symptômes cognitifs et négatifs. Les doses plus importantes, impliquant au moins partiellement un blocage des récepteurs $D_{2}$, sont actives dans les modèles prédictifs de propriétés anti-positives. L'ensemble de ces effets est exprimé en l'absence relative d'effets SEP moteurs. Ces observations, ainsi que la très bonne tolérance du S 33138 et son profil pharmacocinétique favorable, ont encouragé son passage en clinique. Les études de Phase I chez des volontaires sains ont confirmé la bonne tolérance du S 33138. D'ailleurs, les études en PET (positron emission tomography) ont montré une bonne imprégnation des récepteurs $D_{2}$ centraux. Bien qu'il n'y ait pas de ligand PET pour marquer les récepteurs $D_{3}$, ces résultats permettent une estimation des doses les plus faibles requises pour occuper préférentiellement les sites $D_{3}$. En 2005, les études de Phase II seront lancées chez les patients schizophrènes. Ainsi, 15 ans après le clonage des récepteurs $D_{3}$ par une équipe de l'Inserm [46], et à la suite des efforts importants de recherche de la part de nombreuses équipes dans le monde, la première évaluation clinique d'un antagoniste optimisé des récepteurs $D_{3}$ pour le traitement de la schizophrénie va être entreprise. Ce délai ne montre en rien un manque d'efficacité de la Recherche et Découverte, ni d'éventuelles tergiversations avant le lancement d'une démarche clinique; il témoigne tout simplement de l'énorme défi que représente la détermina- 
tion du rôle fonctionnel, et de la signification thérapeutique des nouvelles cibles, ainsi que de la grande difficulté rencontrée dans l'identification de médicaments candidats compatibles avec un développement clinique.

\section{Utilisation des antagonistes $D_{3}$ dans les domaines thérapeutiques autres que la schizophrénie}

Avant de conclure cet article, il faut rapidement évoquer l'intérêt thérapeutique des antagonistes $D_{3}$ (optimisé ou sélectif) dans le traitement de maladies autres que la schizophrénie.

\section{Traitement de l'abus de drogues,} pertinence aux états psychotiques

De nombreuses études ont récemment démontré le grand intérêt des antagonistes $D_{3}$ dans le traitement des conduites addictives et de récidives associées à la consommation de diverses drogues comme la nicotine, les opiacés et surtout la cocaïne $[47,48]$. Comme cela est mentionné ci-dessus, le taux de récepteurs limbiques $D_{3}$ est élevé chez les cocaïnomanes. Un contrôle de l'abus de cocaïne par les antagonistes $D_{3}$ est d'autant plus intéressant que l'utilisation répétée de la cocaïne est associée à un risque accru de développer une schizophrénie. Effectivement, l'exposition répétée à la cocaïne entraîne une sensibilisation de la transmission mésolimbique dopaminergique analogue à l'hypersensibilité à l'origine des états psychotiques [21, 22]. D'ailleurs, les patients psychotiques abusent souvent de cocaïne et autres drogues. En conséquence, la notion des antagonistes $D_{3}$ «doublement » actifs contre l'abus de drogue et la schizophrénie est très séduisante.

\section{Autres maladies}

Les propriétés procognitives des antagonistes $D_{3}[35-$ 37] pourraient avoir une application plus étendue dans le contrôle des atteintes cognitives associées à d'autres maladies comme la dépression, la maladie d'Alzheimer (caractérisée par des déficits cholinergiques) et la maladie de Parkinson. Concernant cette dernière, l'influence facilitatrice des antagonistes $D_{3}$ sur la fonction motrice pourrait même améliorer les symptômes moteurs des malades $[17,20]$.

\section{Conclusions}

La schizophrénie représente toujours un grand défi thérapeutique. Sans pour autant négliger d'autres pistes de recherche, les mécanismes dopaminergiques, les seuls validés cliniquement, gardent toute leur perti- nence. Un blocage des récepteurs $D_{3}$, soit sélectif, soit préférentiel versus $D_{2}$, représente un concept innovant pour améliorer le traitement des états psychotiques, surtout les symptômes cognitifs qui sont mal contrôlés par les médicaments disponibles actuellement. Le premier composé antagoniste $D_{3}$ optimisé, S 33138, va bientôt faire l'objet d'études cliniques d'efficacité Phase II. Les résultats de ces études devraient nous fournir les données concrètes sur l'intérêt des récepteurs $D_{3}$ en tant que cibles thérapeutiques permettant d'améliorer le traitement de cette maladie. $\diamond$

\section{REMERCIEMENTS}

L'auteur tient à remercier G. Lavielle, P.F. Pénelaud, E. Canet, M. Brocco, A. Gobert et J.M. Rivet pour les commentaires utiles sur ce manuscrit, et B. Di Cara pour la préparation des graphiques.

\section{SUMMARY}

Dopamine $D_{3}$ receptors as a novel target for improving the treatment of schizophrenia Schizophrenia is a complex and serious disorder which affects some $0.5-1.0 \%$ of the population. The disease generally begins in adolescence. This early onset, together with the progressive and often irreversible nature of schizophrenia, account for its high social cost. Positive symptoms, such as hallucinations, are generally wellcontrolled by antipsychotics, whereas cognitive and deficit symptoms are poorly-treated. All antipsychotic agents, irrespective of their overall receptor-binding profiles, interact with dopaminergic mechanisms that are known to be perturbed in schizophrenic patients. Dopamine exerts its actions via five classes of receptor, offering a broad palette of targets for the conception of novel antipsychotic agents. The present article focuses on the relevance of dopamine $D_{3}$ receptors to the aetiology and treatment of schizophrenia. Experimental studies suggest that, as compared to other drugs, antipsychotic agents which preferentially block $D_{3}$ receptors may possess therapeutic advantages, notably in the control of cognitive symptoms. The first clinical studies for the evaluation of this hypothesis have recently got underway. $\diamond$

\section{RÉFÉRENCES}

1. Hardy-Baylé MC. Schizophrénie: symptômes et diagnostic. Med Ther 1996; 2: 613-20.

2. Clervoy P, Corcos M. Épidemiologie et histoire naturelle de la schizophrénie. Rev Prat 2002; 52: 1177-82.

3. Harvey PD, Keefe RS. Studies of cognitive change in patients with schizophrenia following novel antipsychotic treatment. Am J Psychiatry 2001; 158: 176-84.

4. Meltzer Hy. Cognitive factors in schizophrenia: causes, impact, and treatment. CNS Spectr 2004; 9: 15-24.

5. Davis JM, Chen N, Glick ID. A meta-analysis of the efficacy of second- 
generation antipsychotics. Arch Gen Psychiatry 2003; 60 : 553-64.

6. Houy-Durand $\varepsilon$, Thibaut F. Traitements des schizophrénies. Rev Prat 2002; $52: 1213-20$

7. Krebs MO. Hypothèses génétiques de la schizophrénie. Rev Prat 2002; 52 : 1208-11.

8. Harrison PJ, Weinberger DR. Schizophrenia genes, gene expression, and neuropathology: on the matter of their convergence. Mol Psychiatry $2005 ; 10: 40-68$.

9. Niznikiewicz MA, Kubicki M, Shenton ME. Recent structural and functiona imaging findings in schizophrenia. Curr Opin Psychiatry 2003; 16: 123-47.

10. Abi-Dargham A, Laruelle M. Mechanisms of action of second generation antipsychotic drugs in schizophrenia: insights from brain imaging studies. Eur Psychiatry 2005; 20: 15-27.

11. Geyer MA, Ellenbroek B. Animal behavior models of the mechanisms underlying antipsychotic atypicality. Prog Neuropsychopharmacol 2003; 27: 1071-9.

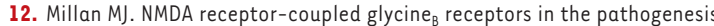
and treatment of schizophrenia: a critical review. Curr Drug Targets 2002; 1: 191-213.

13. Dean $B$, Scarr $\varepsilon$. Antipsychotic drugs: evolving mechanisms of action with improved therapeutic benefits. Curr Drug Targets 2004; 3: 217-25.

14. Millan MJ, Brocco M, Gobert A, et al. Contrasting mechanisms of action and sensitivity to antipsychotics of phencyclidine versus amphetamine: importance of nucleus accumbens $5-\mathrm{HT}_{2 \mathrm{~A}}$ sites for $\mathrm{PCP}$-induced locomotion in the rat. Eur J Neurosci 1999; 11: 4419-32.

15. Meltzer HY, Li Z, Kaneda Y, Ichikawa J. Serotonin receptors: their key role in drugs to treat schizophrenia. Prog Neuropsychopharmacol Biol Psychiatry 2003; 27: 1159-72.

16. Meltzer HY, Arvanitis L, Bauer D, et al. Placebo-controlled evaluation of four novel compounds for the treatment of schizophrenia and schizoaffective disorder. Am J Psychiatry 2004; 161: 975-84.

17. Svensson TH. Alpha-adrenoceptor modulation hypothesis of antipsychotic atypicality. Prog Neuropsychopharmacol Biol Psychiatry 2003; $27: 1145-58$

18. Kapur S, Mamo D. Half a century of antipsychotics and still a central role for dopamine $D_{2}$ receptors. Prog Neuropsychopharmacol Biol Psychiatry 2003; $27: 1081-90$.

19. Joyce JN. Dopamine $D_{3}$ receptor as a therapeutic target for antipsychotic and antiparkinsonian drugs. Pharmacol Ther 2001; 90: 231-59.

20. McGowan S, Lawrence AD, Sales T, et al. Presynaptic dopaminergic dysfunction in schizophrenia: a positron emission tomographic ${ }^{18} \mathrm{~F}$ fluorodopa study. Arch Gen Psychiatry 2004; 61: 134-42.

21. Tsapakis EM, Guillin 0, Murray RM. Does dopamine sensitisation underlie the association between schizophrenia and drug abuse? Curr Opin Psychiatry 2003; 16: S45-52.

22. Richtand NM, Woods SC, Berger P, Strakowski SM. D dopamine receptor, behavioral sensitization, and psychosis. Neurosci Biobehav Rev 2001; 25 : 427-43.

23. Hill K, Mann L, Laws KR, et al. Hypofrontality in schizophrenia: a metaanalysis of functional imaging studies. Acta Psychiatr Scand 2004; 110: 243-56.

24. Millan MJ, Seguin L, Gobert $A$, et al. The role of dopamine $D_{3}$ compared with $D_{2}$ receptors in the control of locomotor activity: a combined behavioural and neurochemical analysis with novel, selective antagonists in rats. Psychopharmacology 2004; 174: 341-57.

25. Holmes A, Lachowicz JE, Sibley DR. Phenotypic analysis of dopamine receptor knockout mice: recent insights into the functional specificity of dopamine receptor subtypes. Neuropharmacology 2004; 47: 1117-34.

26. Neve KA, Seamans JK, Trantham-Davidson H. Dopamine receptor signalling. J Recept Signal Transduct Res 2004; 24: 165-205.

27. Steele TD, Hodges DB, Levesque TR, Locke KW. $D_{1}$ agonist dihydrexidine releases acetylcholine and improves cognitive performance in rats. Pharmacol Biochem Behav 1997; 58: 477-83.

28. Swainston Harrison T, Perry CM. Aripiprazole: a review of its use in schizophrenia and schizoaffective disorder. Drugs 2004; 64: 1715-36.

29. Millan MJ, Dekeyne A, Rivet JM, et al. S33084, a novel, potent, selective, and competitive antagonist at dopamine $\mathrm{D}_{3}$-receptors. II. Functional and behavioural profile compared with GR218,231 and L741,626. J Pharmacol Exp Ther 2000; 293: 1063-73.

30. Silverdale MA, Nicholson SL, Ravenscroft $P$, et al. Selective blockade of $D_{3}$ dopamine receptors enhances the anti-parkinsonian properties of ropinirole and levodopa in the MPTP-lesioned primate. Exp Neurol 2004; 188: $128-38$
31. Millan MJ. The role of monoamines in the actions of established and «novel» antidepressant agents: a critical review. Eur J Pharmacol 2004; 500: 371-84.

32. Reavill C, Taylor SG, Wood MD, et al. Pharmacological actions of a novel, high-affinity, and selective human dopamine $D_{3}$ receptor antagonist, SB277011-A. J Pharmacol Exp Ther 2000; 294: 1154-65.

33. Jongen-Relo AL, Drescher KU, Teschendorf HJ, et al. Effects of dopamine $D_{3}$ receptor antagonists and antipsychotic drugs on the disruption of huddling behavior by dopamine agonists. Am Soc Neurosci 2004; 29: 350.2 (abstract).

34. Robertson GS, Lee CJ, Sridhar K, et al. Clozapine-, but not haloperidol-, induced increases in FosB-like immunoreactivity are completely blocked in the striatum of mice lacking $D_{3}$ dopamine receptors. Eur J Neurosci $2004 ; 20: 3189-94$.

35. Glickstein SB, Desteno DA, Hof PR, Schmauss C. Mice lacking dopamine $D_{2}$ and $D_{3}$ receptors exhibit differential activation of prefrontal cortical neurons during tasks requiring attention. Cereb Cortex 2004 (10 novembre, online).

36. Dekeyne A, Di Cara B, Gobert A, Millan MJ. Blockade of dopamine D receptors enhances frontocortical cholinergic transmission and cognitive function in rats. Am Soc Neurosci 2004; 29: 776.4 (abstract).

37. Lacroix LP, Hows ME, Shah AJ, et al. Selective antagonism at dopamine $D_{3}$ receptors enhances monoaminergic and cholinergic neurotransmission in the rat anterior cingulate cortex. Neuropsychopharmacology 2003; 28 839-49.

38. Leriche L, Diaz J, Sokoloff P. Dopamine and glutamate dysfunctions in schizophrenia: role of the dopamine $D_{3}$ receptor. Neurotox Res $2004 ; 6$ : $63-72$

39. Gurevich EV, Bordelon Y, Shapiro RM, et al. Mesolimbic dopamine $D_{3}$ receptors and use of antipsychotics in patients with schizophrenia. A postmortem study. Arch Gen Psychiatry 1997; 54: 225-32.

40. Dubertret C, Gorwood P, Ades J, et al. Meta-analysis of DRD3 and schizophrenia. Am J Med Genet 1998; 81: 318-22.

41. Jonsson EG, Flyckt L, Burgert $\varepsilon$, et al. Dopamine D3 receptor gene Ser9Gly variant and schizophrenia: association study and meta-analysis. Psychiatr Genet 2003; 13: 1-12.

42. Luedtke RR, Mach RH. Progress in developing $D_{3}$ dopamine receptor ligands as potential therapeutic agents for neurological and neuropsychiatric disorders. Curr Pharmacol Design 2003; 9: 643-71.

43. Dubuffet T, Newman-Tancredi A, Cussac D, et al. Novel benzopyrano [3,4c] pyrrole derivatives as potent and selective dopamine $D_{3}$ receptor antagonist. Bioorg Med Chem Lett 1999; 9: 2059-64.

44. Brocco M, Gobert A, Dekeyne A, et al. S33138, a novel and preferentia antagonist at dopamine $D_{3}$ receptors: functional profile in rodent models of the control of negative-cognitive symptoms of schizophrenia. Int J Neuropsychopharmacol 2004; 7 (suppl 1): S265 (abstract).

45. Marder SR, Fenton W. Measurement and treatment research to improve cognition in schizophrenia: NIMH MATRICS initiative to support the development of agents for improving cognition in schizophrenia. Schizophrenia Res 2004; 72: 5-9.

46. Sokoloff $P$, Giros $B$, Martres MP, et al. Molecular cloning and characterization of a novel dopamine receptor $\left(D_{3}\right)$ as a target for neuroleptics. Nature 1990; 347: 146-51.

47. Xi ZX, Gilbert J, Campos AC, et al. Blockade of mesolimbic dopamine $D_{3}$ receptors inhibits stress-induced reinstatement of cocaine-seeking in rats. Psychopharmacology 2004; 176: 57-65.

48. Le Foll $B$, Francès $H$, Diaz J, et al. Role of the dopamine $D_{3}$ receptor in reactivity to cocaine-associated cues in mice. Eur J Neurosci 2002; 15 : 2016-26.

\section{TIRÉS À PART}

M.J. Millan 